NASA Technical Memorandum 102618

\title{
DIGITAL-FLUTTER-SUPPRESSION-SYSTEM INVESTIGATIONS FOR THE ACTIVE FLEXIBLE WING WIND-TUNNEL MODEL
}

Boyd Perry III, Vivek Mukhopadhyay, Sherwood T. Hoadley, Stanley R. Cole, Carey S. Buttrill, and Jacob A. Houck

March 1990
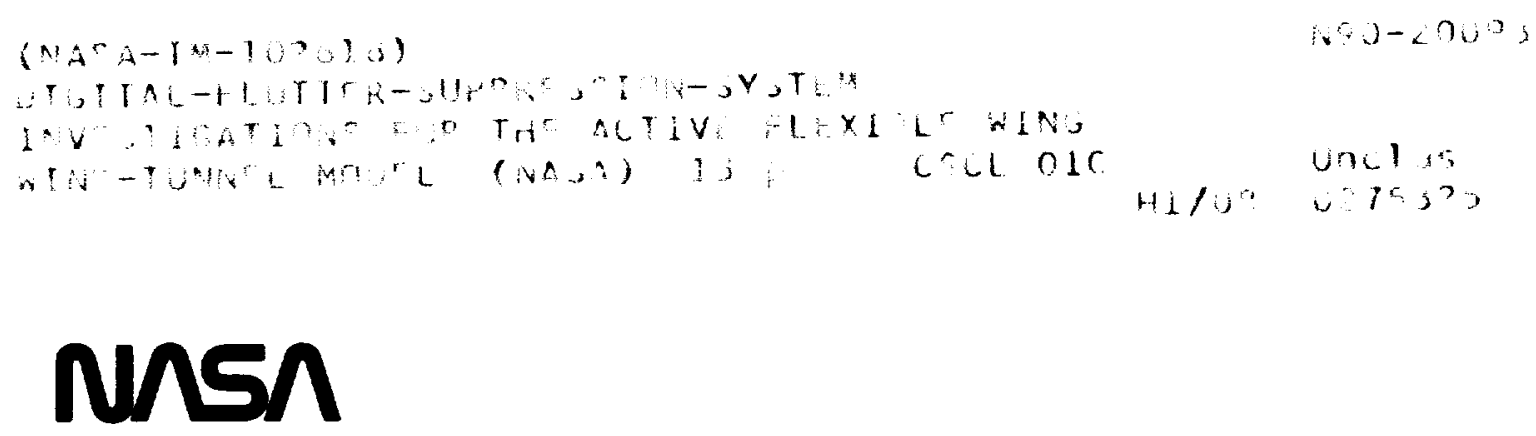

National Aeronautics and

Space Administration

Langley Research Center

Hampton, Virginia 23665-5225 

Boyd Perry III, Vivek Mukhopadhyay ${ }^{\dagger}$, Sherwood Tiffany Hoadley ${ }^{\dagger}$, Stanley R. Cole ${ }^{* *}$, Carey S. Buttrill ${ }^{*}$, and Jacob A. Houck ${ }^{\dagger}$

NASA Langley Research Center

Hampton, Virginia 23665-5225

\begin{abstract}
Ahstract
Active flutter suppression control laws were designed, implemented, and tested on an aeroelastically-scaled windtunnel model in the NASA Langley Transonic Dynamics Tunnel. One of the control laws was successful in stabilizing the model while the dynamic pressure was increased to 24 percent greater than the measured openloop flutter boundary. Other accomplishments included the design, implementation, and successful operation of a one-of-a-kind digital controller, the design and use of two simulation methods to support the project, and the development and successful use of a methodology for online controller performance evaluation.
\end{abstract}

\section{Introduction}

The Active Flexible Wing (AFW) Program (ref. 1) is a cooperative effort between the NASA Langley Research Center and Rockwell International Corporation. The program objective is the validation of analysis and synthesis methodologies through the development of realtime digital multi-input multi-output (MIMO) control laws for a sophisticated aeroelastic wind-tunnel model. This model was tested in the Langley Transonic Dynamics Tunnel during the Fall of 1989.

One of the active control concepts being investigated in the AFW Program is flutter suppression (FS). The design goal for FS control laws was to increase the passive flutter dynamic pressure 30 percent. In order to meet this design goal, the FS control laws had to be capable of suppressing both symmetric and antisymmetric flutter instabilities simultaneously. In addition the FS control laws had to be practical and of low-order, robust, and capable of real-time execution.

The purpose of this paper is to present an overvicw of the FS portion of the AFW Program, emphasizing the various activities which contributed to the overall success of the recent wind tunnel test.

* Member AIAA

** Senior Member, AIAA

$\dagger$ Associate Fellow, AIAA

\section{Test_Anparatus}

\section{Wind Tunnel}

The AFW wind-tunnel model was tested in the Langley Transonic Dynamics Tunnel (TDT) (ref. 2). The TDT is a closed-circuit, continuous-flow wind tunnel capable of testing at stagnation pressures from near zero to atmospheric and over a Mach number range from zero to 1.2. The test section of the TDT is $16.0 \mathrm{ft}$. square with cropped corners. The TDT has several model support options, which include wall mounting, sting mounting, and free-flying cable mounting.

A feature of the TDT which is particularly useful for aeroelastic testing is a group of four bypass valves, which when opened, connect the return leg of the tunnel aft of the drive motor directly to the plenum surrounding the slotted test section. In the event of a model instability, such as flutter, these quick-actuating valves are opened. This causes a rapid reduction in the test section Mach number and dynamic pressure which may result in stabilizing the model. During the recent AFW test, instrumentation on the model was monitored using electronic equipment that automatically commanded the bypass valves to open when model response exceeded a predetermined criteria of load amplitude and frequency.

The TDT is capable of testing with either an air or a heavy gas (Freon-12) test medium. The model used in the present study was tested in air.

\section{Wind-Tunnel_Model}

Figure 1 shows the AFW model mounted in the TDT during the most recent test entry. The AFW wind-tunnel model is a full-span, aeroelastically-scaled representation of a fighter aircraft concept. It has a moderate aspect ratio wing with a span of 8.67 feet. The model was supported on the wind-tunnel test section centerline by a sting mount specifically constructed for testing the AFW model. This sting utilizes an internal ballbearing arrangement to allow the model freedom to roll about the sting axis. The fuselage is connected to the sting through a pivot arrangement so that the model can be remotely 
pitched from approximately -1.5 degrees to +13.5 degrees angle of attack.

Structure. The fuselage of the model is designed to be rigid. It is constructed from aluminum stringers and bulkheads with a fiberglass skin providing the appropriate external shape. The wings of the model are constructed from an aluminum honeycomb core co-cured with tailored plies of a graphite/epoxy composite material. The plies were oriented to permit desired amounts of bending and twist under acrodynamic loads. The surfaces of the graphite/epoxy material were covered by a semi-rigid polyurethane foam to provide the airfoil shape without significantly affecting the wing stiffness.

Control_Surfaces. The model has two leadingedge and two trailing-edge control surfaces on each wing panel. These control surfaces are constructed of polyurethane foam cores with graphite/epoxy skins. Each control surface has a chord of 25 percent of the local wing chord and a span of 28 percent of the wing semispan. The control surfaces are connected to the wing by hinge-linemounted, vane-type rotary actuators powered by an onboard hydraulic system. The actuators are connected to the wing structure by cylindrical rods which are fitted in titanium inserts in the wing. This arrangement is designed to provide the shear and torsion requirements placed on the wing-to-control surface connections and yet allow for bending freedom of the wing. This also minimizes the contribution of the control surfaces to the wing stiffness. Deflection limits are imposed on the various control surfaces to avoid exceeding hinge-moment and wing-load limitations.

Instrumentation. The AFW model was instrumented with a six-component force-and-moment balance, accelerometers, strain-gage bridges, rotary variable differential transformers (to measure controlsurface deflection angles), a roll potentiometer, and a rollrate gyro.

Tip Ballast Store. The AFW model was modified for the recent wind-tunnel test so that flutter would occur within the operating envelope of the TDT. This modification consisted of adding a tip ballast store to each wing panel. A drawing of the tip ballast store is shown in figure 2. The store is basically a thin, hollow aluminum tube with distributed internal ballast to lower the basic wing flutter boundary to a desired dynamic pressure range. Additionally, the store provides a model safety feature. Instead of a hard attachment, the store is connected to the wing by a pitch-pivot mechanism. The pivot allows freedom for the tip store to pitch relative to the wing surface. When testing for flutter, an internal hydraulic brake held the store to prevent such rotation. In the event of a flutter instability, this brake was relcased. In the released configuration, the pitch stiffness of the store is provided by a spring element internal to the store as shown in the figure. The reduced pitch stiffness of the spring element (as compared to the pitch stiffness of the hydraulic brake arrangement) significantly increases the frequency of the first torsion mode of the wing store assembly. This behavior is related to the concept of the decoupler pylon as discussed in reference 3. The raised torsional frequency leads to a significant increase in the model's flutter dynamic pressure which quickly suppressed the motion of the model on numerous occasions during the test.

\section{Predicted Open-Loop Elutter Characteristics}

Figure 3 contains a plot of the TDT operating envelope in air and the analytical open-loop flutter-boundary predictions. The heavy solid line is the operating envelope and the steep slope on the left represents operating at atmospheric conditions in the tunnel. It was along this portion of the envelope that all flutter suppression tests were conducted. For tests conducted in air, the maximum possible dynamic pressure (with no model blockage) is 325 pounds per square foot (psf).

For the symmetric flutter analyses, doublet lattice aerodynamics were used with eight flexible modes obtained from a NASTRAN analysis; for antisymmetric analyses, seven flexible modes were used. In each case, modal frequencies were modified to match values measured during a ground vibration test.

The solid lines in the figure represent the predicted symmetric and antisymmetric flutter boundaries for the tip ballast store in its coupled configuration; the dashed lines represent the boundaries for the decoupled configuration. Flutter frequencies are indicated in parentheses in the figure. Along the atmospheric line, the open-loop flutter dynamic pressures are 248 psf and 252 psf. It can be seen that the design goal of increasing the flutter dynamic pressure by 30 percent was defined by the capabilities of the wind tunnel.

\section{Elutter_Suppression Control Law Desiens}

This section of the paper addresses the various aspects of the design of the flutter suppression controls laws. Three separate control laws were designed, implemented, and tested.

\section{Design Ohiective and Goals}

The design objective was to develop low-order robust digital flutter-suppression (FS) control laws which would simultaneously suppress symmetric and antisymmetric flutter while satisfying the following: stability over the entire test dynamic pressure range; singular-value-based MIMO multiplicative stability margins corresponding to $\pm 6 \mathrm{db}$ and \pm 45 degrees at both the plant input and plant output; and acceptable control surface activity. The design goal was to increase the lowest open-loop flutter dynamic pressure by 30 percent. 
Elutter Sunoression Scheme

It was assumed, for this wind-tunnel model, that there was no coupling between the symmetric and antisymmetric modes of motion. Therefore, symmetric and antisymmetric flutter suppression control laws were designed separately. A block diagram illustrating the scheme for combining both control laws is shown in figure 4. Signals from the right and left wing sensors (accelerometers) are sampled, digitized, and then summed and differenced to form symmetric and antisymmetric signals. These signals are then processed by their respective control laws and recombined to form right and left actuator commands. Finally, these commands are converted to analog signals and sent to the wind-tunnel model.

\section{Design Procedures}

Procedure 1 - LOG Design and Order Beduction. This procedure uses the plant state-space model and weight and noise covariance matrices as input to create a model-based full-order Linear-QuadraticGaussian (LQG) design. A minimum energy optimal regulator was designed with zero state weighting and unit control weighting matrices. The model-based minimum variance state estimator was designed with 0.000001 radians fictitious plant-input noise, one-inch-per-second gust input noise and $0.01 \mathrm{~g}$ (gravitational acceleration) measurement noise. Based on non-minimal phase transmission zeros contained in the state-space model and many poorly controllable and observable states, the noise intensities were chosen after a few trials so as to produce a low-gain LQG control law with stable poles. The plantinput noise was used to improve the robustness of the LQG control law at the plant input.

The next step in the procedure was control law order reduction. In actual practice, the model-based full-order LQG control law was reduced by balanced realization (ref. 4) followed by modal residualization. When a stable reduced-order control law was found, the full-order and the reduced-order control laws were analyzed and the singular values, frequency responses and root-mean-square ( $\mathrm{rms}$ ) responses were compared. Although a lower-order stable control law could be found, the tenth-order control law was chosen since its performance was close to the performance of the full-order LQG control law.

The final step was to discretize the final continuous control law at 200 samples per second using Tustin transforms. This control law uses two pairs of sensors and two pairs of control surfaces.

Procedure 2 - Classical Design. This procedure makes use of classical root-locus and Nyquist techniques and relies on a fundamental physical understanding of the flutter mechanism. A unique aspect of the design is that it takes advantage of an effective pole-zero cancellation associated with the first wing torsion mode at low dynamic pressure, and pole-zero migrations with variations in dynamic pressure. Thirdorder FS control laws (per symmetry) were obtained in the continuous domain and then implemented digitally. This control law uses one pair of sensors and two pairs of control surfaces.

Procedure 3 - Synthesized Modal Velocity This procedure chooses parameters which define linear combinations (blending) of available accelerometers to create sensors that observe the modal velocities of the critical flutter modes and then distributes control commands to available actuators. The goal of the controller is to prevent flutter by adding damping to the critical flutter mode while satisfying control power, hinge moment, and other practical constraints. This design approach extends the work defined in reference 5 by including design variables to distribute the control commands to the available control surface actuators and the incorporation of inequality constraints. Fifth-order FS control laws (per symmetry) were obtained in the continuous domain and then implemented digitally. This control law uses four pairs of sensors and two pairs of control surfaces.

\section{Predicted ES Control Law Performance}

Figure 5(a) contains predicted symmetric flutter root loci (as a function of dynamic pressure) for both open- and closed-loop conditions; figure 5(b) contains predicted antisymmetric flutter root loci. Both closed-loop calculations are based on the control law designed using procedure 1 (Control Law 1). As indicated by the insets in the figures, the goal of increasing the lowest open-loop flutter dynamic pressure by 30 percent was met analytically.

\section{Digital Controller}

One of the primary objectives of the AFW Program is to gain practical experience in designing, fabricating, and implementing a real-time MIMO digital controller and in developing the hardware interface between the controller and the wind-tunnel model. Design specifications required that the controller have the capability of receiving and sending both analog and discrete signals. Furthermore, it had to sample data and execute control laws 200 times per second. To meet these requirements with reasonable resources, a SUN $3 / 160$ workstation driven by a Unix operating system was selected as the "shell" of the digital controller.

The digital controller controls the wind-tunnel model by digitizing the incoming sensor signals, processing the currently-implemented control law, and then providing the appropriate control-surface actuator commands to execute these laws. Additional capabilities of the digital controller include the manual positioning of the control surfaces, the calculation and application of excitation signals to various control surfaces, and the recording, transferring, and storing of digitized signals. 


\section{Hardware Components}

The hardware components of the digital controller are shown schematically in figure 6 . The components include a host computer, a digital signal processor board, an array processor board, two analog-to-digital, and two digital-to-analog conversion boards.

The host computer is a SUN $3 / 160$ workstation. It provides user interface to the digital signal processor board, intializes user options, control law arrays, control parameters, and excitation definitions. The host downloads signal-processor software, and determines and downloads the array-processor command code to implement a currently-selected control law. It allows realtime changes in mode of operation, gains, excitation amplitudes, and the control surfaces to be used. The host controls the saving of the digitized data to external files and tapes and provides the display of important parameters such as control-surface deflections, errors between commanded and actual deflections, overall control-law gain, and switch selections.

The digital signal processor (DSP) is a Challenger board manufactured by SKY Computers, Inc. and is composed primarily of two TMS 32020 microcomputers and $64 \mathrm{~K}$ integer words ( 2 bytes) of memory. This board is referred to as "the controller" because it controls, directs, and sequences all the real-time activities and tasks. It controls all the real-time processing of analog input and output signals; it controls control-law execution by sending commands to the array processor cpu; it provides the interface to the user control panel lights and switches; and it sets switches (software flags) for the host computer which specify when blocks of data can be stored and transferred.

The array processor (AP) is a SKY Warrior I board with 16 Mbytes of memory. It performs all the floating-point processing required for control law execution, including unit conversions, scaling, and all matrix computations.

The analog-digital conversion boards consist of two DT1401 analog-to-digital converter boards and two DT-1406 digital-to-analog converter boards manufactured by Data Translation, Inc. They perform all the analog-to-digital and digital-to-analog conversions.

The user control panel, designed and built in-house by NASA, provides the real-time interface to "the controller". It allows real-time selection of certain options via lighted switches and provides real-time status of various control parameters through status display lights. These switches are simulated in the host interface software for use with the simulator and as a backup.

\section{Software}

All software was written in the high level C programming language, except for the commands required to perform the actual calculations on the array processor. Operation code command blocks were generated for these.
There are three primary host/interface programs, a controller program written in C, but with its own compiler code, and control law execution code written using the array processor command language.

A generic form of the control-law function was identified such that one set of software would accommodate a given control law while imposing minimal constraints on the control-law designers. The generic controller structure allows the designers the choice of sensors with the option to blend them, freedom of controller order (with upper limits), scheduling with dynamic pressure of controller parameters, and the choice of control surfaces with the option to distribute control-law outputs to different actuators. The generic form of the control-law function allows for changes in a design to be implemented easily and reliably.

\section{Technical Difficulties}

There were four primary difficulties encountered in designing, coding, and assembling this one-of-a-kind digital controller

The first was that the real-time controller had to operate at $200 \mathrm{~Hz}$ within a Unix-based operating system which operates at $60 \mathrm{~Hz}$. This necessitated obtaining, from the manufacturer of the DSP, code that gave control of the data bus to the DSP computer, which operated as fast as calculations would permit.

The second difficulty was that no two host software codes could communicate with either the DSP memory or the AP memory simultaneously. Since the primary functions of the host computer fell into three categories, three different software packages were developed. This challenge was met by using the DSP to pass information between the various host programs.

The third difficulty resulted from the fact that the DSP was capable of performing integer arithmetic only. It had no floating point registers. This was solved by doing the following: (1) performing most floating point arithmetic on the AP, which entailed transferring data and command codes to the AP; and (2) performing the remainder on the DSP, which entailed writing code for approximating floating point calculations using integer arithmetic on the DSP. It would have been preferable to have had a digital signal processor which was capable of some floating point arithmetic.

The fourth difficulty was that the analog-digital and digital-analog boards only generated 12 bits of resolution. This not only caused some voltage resolution loss, but also necessitated careful handling of sign extensions and truncations from and to 16-bit integer data by the DSP. These operations used a significant portion of the 5 millisecond time budget allowed by the $200 \mathrm{~Hz}$ sampling rate. It also required special code to implement voltage limiters on signals which required comparisons of 12-bit signed data with 16-bit compare registers. It would have 
been highly desirable to have had 16-bit data conversion boards.

\section{Simulation}

Two varieties of simulation were employed to support preparations for the wind-tunnel test: a hot bench simulation and a comprehensive nonlinear batch simulation.

\section{Hot Bench Simulation}

Open-loop flutter analytical results for the model indicated that the onset of flutter would be very rapid. At test conditions of 0.5 Mach number and 300 psf, the predicted time-to-double amplitude for flutter was about 0.12 seconds. For closed-loop testing above the open-loop flutter boundary, any digital control system failure might result in very rapid loss of the model before the tip-ballast store brake could be released or before the tunnel bypass valve openings effectively reduced tunnel conditions. Also, the effectiveness of the tip ballast store as a flutter stopper was unknown prior to the test. Because there was a lot of concern for the safety of the wind-tunnel model, it was felt essential to do a pretest verification of the digital controller to gain confidence that the systems functioned properly. This verification was performed by coupling the digital controller to a computer simulation of the model being tested in the tunnel. Because the computer simulation sends signals to and receives signals from the hardware setup, it is referred to as a hot bench simulation (HBS).

The HBS was implemented using the Langley Advanced Real-Time Simulation (ARTS) System described in reference 6. The ARTS consists of two Cyber 175 computers connected to an array of simulation sites by means of a 50-megabit-per-second fiber optic digital data network called Computed Automated Measurement and Control (CAMAC). The CAMAC interface converts Cyber 175 digital signals to analog signals which are sent to the AFW digital controller through the interface electronics shown in figure 6 . In addition the CAMAC site converts the analog signals generated by the AFW digital controller to digital signals to be sent to the Cyber 175. An Adage Graphics Computer, interfaced directly to the Cyber 175, was used to generate a color-coded, threedimensional wireframe outline of the AFW model. The display presents model pitch, roll and yaw, control surface deflections and total model deformation. A blue shadow wireframe of the undeformed model is drawn so that deformations are more easily scen. An example is shown in figure 7 .

HBS Timing. The HBS used an integration step size of 0.0005 seconds but was executed at an update clock rate of $80 \mathrm{~Hz}$. Thus the HBS ran in synchronized real time at 25:1 "slow". The discrete control laws were designed to be executed at $200 \mathrm{~Hz}$ in the digital controller for the wind-tunnel test. When coupled to the HBS, the digital controller was therefore clocked at $8 \mathrm{~Hz}$, or $25: 1$ "slow".

A choice of either $25 \mathrm{~Hz}$ or $100 \mathrm{~Hz}$ analog antialiasing filters was available to the control law designers. Because the digital controller was running 25:1 "slow" for the HBS these filters would not have the same effect as during normal operation. To correct for this problem the antialiasing filters were included in the digital portion of the HBS so their effects could be properly simulated.

HBS Math Model. The HBS math model in its final pretest configuration included 8 symmetric and 7 antisymmetric elastic modes, eight third-order actuator models, 15 unsteady aerodynamic "lag" states, symmetric and antisymmetric turbulence models, and first-order 25 $\mathrm{Hz}$ antialiasing filters on 31 feedback signals. Symmetric or antisymmetric modal dynamics could be bypassed in the HBS, allowing isolated testing of either the antisymmetric or symmetric FS control laws, respectively. The individual right and left actuators were modeled separately using third-order transfer functions derived from measured frequency response data. In general, the right actuator models were different from the corresponding left actuator models. No-load rate limits were applied.

In the HBS, once current-time modal accelerations are calculated from the current-time positions and velocities, an Adams-Bashforth second-order predictor (AB2) is used to predict the velocities at the next time step. The predicted velocities are then used in a trapezoidal integration to generate predicted modal positions.

\section{Nonlinear Batch Simulation}

The HBS was verified by comparing results with a more comprehensive nonlinear batch simulation. The batch simulation also modeled the digital controller and included the effects of computational delay and quantization. The open-loop plant dynamics of the batch simulation were verified by extracting linear models using finite differencing and then comparing the frequency response of these batch-derived linear models with the frequency response of linear design models for all combinations of inputs and outputs.

The batch simulation used a Runge-Kutta second-order predictor-corrector (RK2) formula to integrate all state derivatives. (The RK2 integration is typically more accurate than the $A B 2$, but the RK2 formulation requires two derivative evaluations per time step. The $A B 2$ is a single-pass formula which gives up some gain accuracy for phase accuracy and is typically used in real-time applications.) The integration step of 0.0005 seconds was small enough that batch and HBS results compared favorably.

Figure 8 contains typical results obtained from the comprehensive nonlinear batch simulation. Predicted open- and closed-loop (Control Law 1) step responses of the AFW model at an analysis condition 50 psf above the 
symmetric open-loop flutter boundary are presented. The results indicate that, open-loop, the model is unstable and that, closed-loop, the model is stable. When comparing open- and closed-loop responses, note the change in scales (abscissa and ordinate).

\section{Elutter Testing}

\section{Open-Loop Testing}

The AFW model was tested first with the tip ballast store in the decoupled configuration to clear a safe region within the tunnel operating envelope. The model was then tested with the tip ballast store in the coupled configuration to establish the open-loop flutter dynamic pressure. Along the atmospheric line, the flutter mode with the lowest dynamic pressure was antisymmetric at a dynamic pressure of $220 \mathrm{psf}$ and at a frequency of about 9 Hz. This experimental result differed in two ways from the analytical prediction: (1) antisymmetric flutter occurred first experimentally, while symmetric flutter occurred first analytically; and (2) the experimental flutter frequency was about $3 \mathrm{~Hz}$ lower than the analytical prediction. The experimental open-loop flutter point and the analytical predictions are shown in figure 9 . In reference 7, this subsonic open-loop flutter point and three transonic open-loop flutter points are compared with analytical predictions computed using the CAP-TSD (Computational Aeroelasticity Program - Transonic Small Disturbance) code.

\section{Closed-Loon Testing}

All closed-loop FS testing was performed along the atmospheric tunnel-operating line with the tip ballast store in the coupled configuration. Closed-loop testing was performed in the following manner:

- with the wind-tunnel model open-loop, the tunnel conditions were brought up to a dynamic pressure of 100 to $125 \mathrm{psf}$;

- with the wind-tunnel model open-loop, but with the FS control law operating, a controller performance evaluation scheme for estimating relative stability (using estimates of the singular values of the return difference matrix at the plant input and output) was employed to determine closed-loop stability at that condition;

- if the scheme predicted closed-loop stability, the FS loop was closed and dynamic pressure was increased;

- with the FS loop closed and at regular increasing increments in dynamic pressure, the relative-stability scheme was employed again and rms values of controlsurface deflections and rates were obtainer.
All three control laws were tested, with varying degrees of success. Control Law 1 was able to take the model to its open-loop flutter dynamic pressure before going unstable; Control Law 2 was able to go 52 psf beyond (to attain a 24 percent increase in) the open-loop flutter dynamic pressure; Control Law 3 went unstable 35 psf below the open-loop flutter dynamic pressure. Investigations are currently underway to determine the reasons for the disappointing performances of Control Laws 1 and 3.

Figure 10 contains a typical theoretical-experimental comparison obtained during closed-loop testing below the open-loop flutter boundary. Part (a) contains singular value plots of the return-difference matrix $(I+G H)$ at the plant output; part (b) contains complex determinant plots of the return-difference matrix, also at the plant output. (Here $G$ and $H$ are the plant and control law transfer matrices respectively.) Both are for the symmetric Control Law 1 at 200 psf. At this condition, both analytically and experimentally, the model is open-loop and closed-loop stable.

From the comparison in figure 10 (a) it can be seen that the actual system and the truth-model are qualitatively similar but have some discrepancies. Below $15 \mathrm{~Hz}$, and present in both the maximum and minimum singular values, there is a fairly consistent frequency shift of about $2 \mathrm{~Hz}$. Thus, the frequencies at which the predicted and actual minimum singular values occur are about $9 \mathrm{~Hz}$ and $7 \mathrm{~Hz}$, respectively. In addition the magnitudes of these minimum singular values differ, indicating that the actual symmetric closed-loop system was less robust than it was predicted to be. The plots in figure $10(\mathrm{~b})$ can be interpreted as multiloop Nyquist plots. For the cases shown (FS on, below open-loop flutter) there are no encirclements of the origin, which confirms that the system is both open-loop and closed-loop stable.

All three control laws have in common the use of the trailing-edge-outboard (TEO) pair of control surfaces. For all three, this pair of surfaces was the "work horse," deflecting the most in response to sensor inputs to the control laws. For this reason it was to this pair of surfaces that particular attention was paid during the test to assure that control-surface actuator saturation did not occur. Figure 11 contains plots of the root-mean-square values of commanded TEO control-surface rates as functions of dynamic pressure. Over the range of dynamic pressures it can be seen that there was about a 20 percent difference in the magnitude of commanded rates. These rates were well within the capabilities of the actuators, even at the highest dynamic pressures using only about ten percent of the available rate capability.

\section{Concluding Remarks}

Flutter suppression (FS) tests of the Active Flexible Wing wind-tunnel model were conducted in the NASALangley Transonic Dynamics Tunnel. Three FS control laws were designed, implemented, and tested and one was successful in taking the model 24 percent above its 
lowest open-loop flutter dynamic pressure. Other accomplishments which were essential to the overall success of the program were:

- the design, fabrication, and successful operation of the tip ballast store;

- the design, fabrication, coding, and successful operation of the digital controller in which the FS control laws were executed;

- the design and execution of two simulation methods -. one to check the functionality of the digital controller, the other to aid the control law designers in their task;

- the development and successful operation of a methodology for on-line controller performance evaluation

\section{Acknowledgements}

The success of this wind-tunnel entry was due to the combined efforts of all members of the Active Flexible Wing Team. The authors wish to acknowledge these contributions and thank each individual on the Team. The authors wish especially to thank AFW Team members Martin R. Waszak and S. Srinathkumar (Control Law 2) and William M. Adams and David M. Christhilf (Control Law 2) for providing information on their control laws.

\section{ORIGINAL PAGE \\ BLACK AND WHITE PHOTOGRAPH}

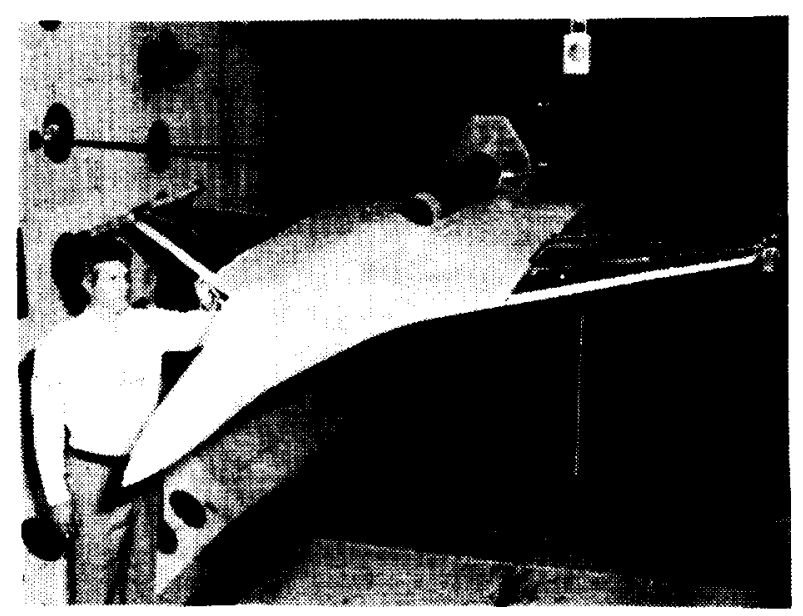

Figure 1. - AFW model mounted in the TDT

\section{References}

1. Noll, Thomas E.; Perry, Boyd III; et al: Aeroservoelastic Wind-Tunnel Investigations Using the Active Flexible Wing Model - Status and Recent Accomplishments. AIAA Paper No. 89-1168-CP. April 1989.

2. Reed, Wilmer H., III: Aeroelasticity Matters: Some Reflections of Two Decades of Testing in the NASA Langley Transonic Dynamics Tunnel. NASA TM-83210, 1981.

3. Reed, W. H., III; Foughner, J. T.; and Runyan, H. L., Jr.: Decoupler Pylon: A Simple, Effective Wing/Store Flutter Suppressor. Joumal of Aircraft, Vol. 17, No. 3, pp. 206-211, March 1980.

4. Laub, S. J.; Heath, M. T.; Paige, C. C.; and Ward, R. C.: Computation of System Balancing Transformations and Other Applications of Simultaneous Diagonalization Algorithms. IEEE Transactions on Automatic Controls, Vol. AC-32, pp. 115-122, February 1987.

5. Johnson, E. H.; Hwang, C.; Joshi, D. S.; Kesler, D. F.; and Harvey, C. A.: Test Demonstration of Digital Control of Wing/Store Flutter. AlAA Joumal of Guidance, Control and Dynamics, Vol. 6, No. 3, pp 176-181, May-June 1983.

6. Crawford, Daniel J.; Cleveland, Jeff I., II; and Staib, Richard O.: The Langley Advanced Real-Time Simulation (ARTS) System Status Report. AIAA Paper No. 88-4595-CP, September 1988.

7. Silva, Walter A.; and Bennett, Robert M.: Using Transonic Small Disturbances Theory For Predicting the Aeroelastic Stability of a Flexible Wind-Tunnel Model. AIAA Paper No. 90-1033-CP, April 1990.

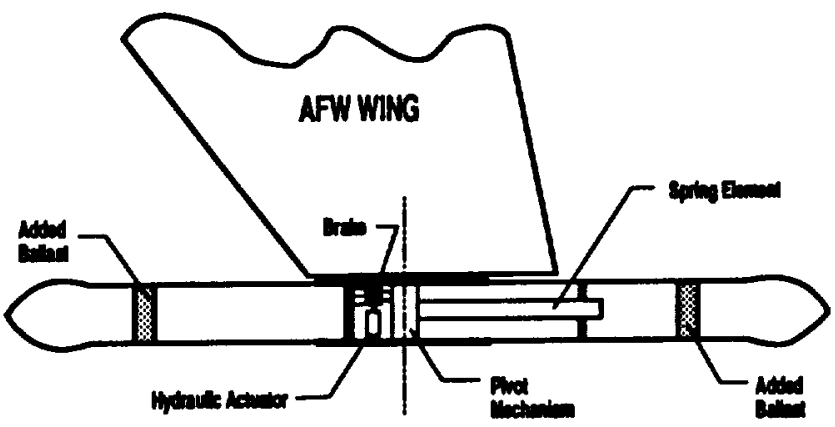

Figure 2. - Tip ballast store of AFW model 


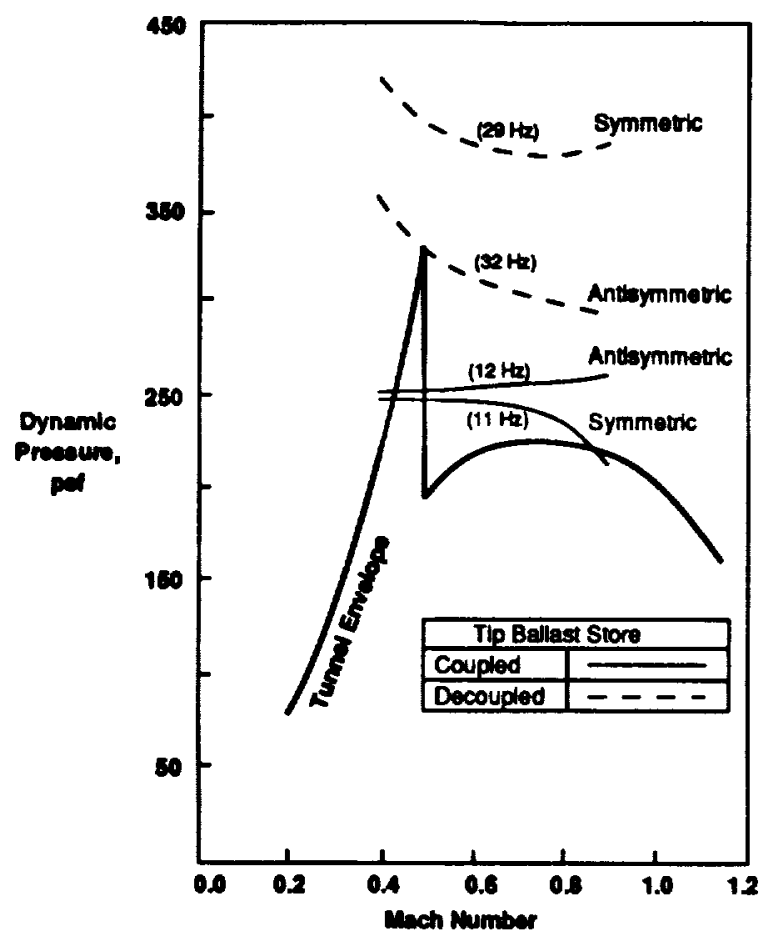

Figure 3. - TDT operating envelope in air with predicted open-loop flutter boundaries

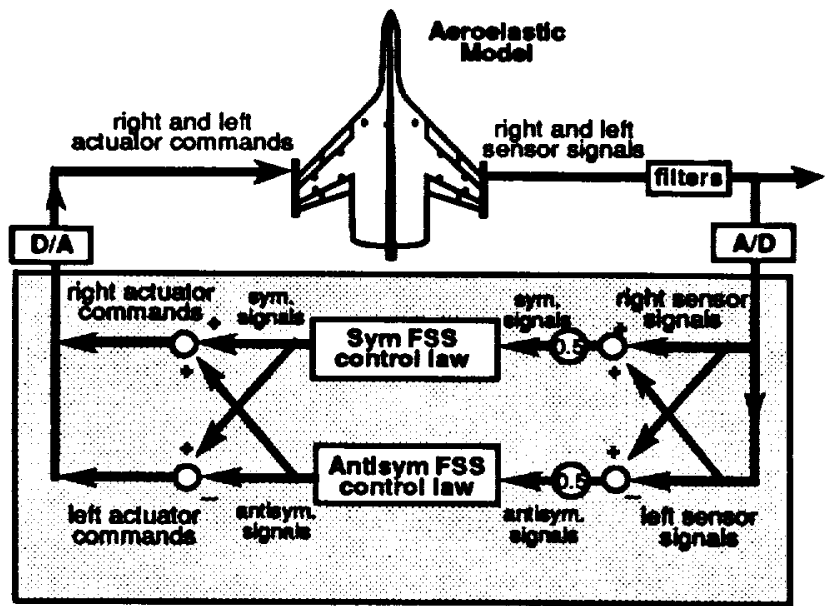

Figure 4. - Scheme for combining symmetric and antisymmetric FS control laws

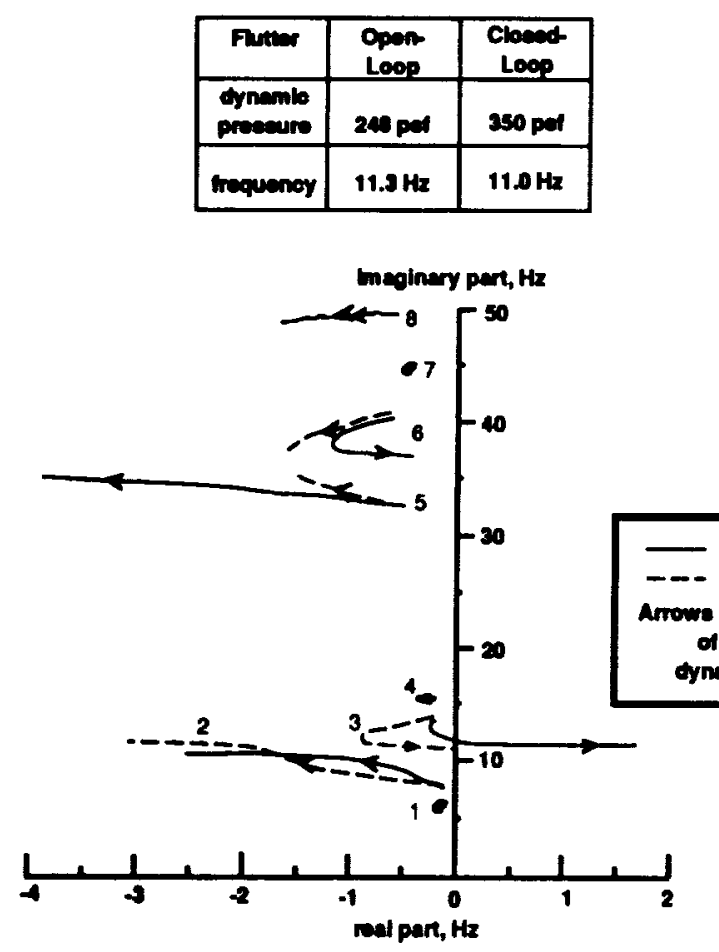

(a) Symmetric

\begin{tabular}{|c|c|c|}
\hline Funer & $\begin{array}{l}\text { Opon } \\
\text { Loes }\end{array}$ & $\begin{array}{l}\text { Clowed } \\
\text { Leep }\end{array}$ \\
\hline Arnamie & $252 \mathrm{pet}$ & 204 pet \\
\hline thequenor & $11.7 \mathrm{ht}$ & $11.2 \mathrm{kt}$ \\
\hline
\end{tabular}

lmaginary part, Hz

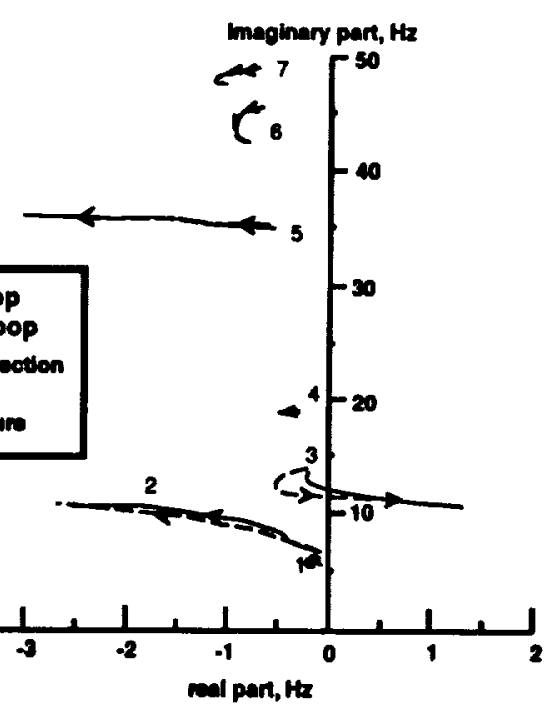

(b) Antisymmetric

Figure 5. - Open- and closed-loop dynamic pressure root loci 


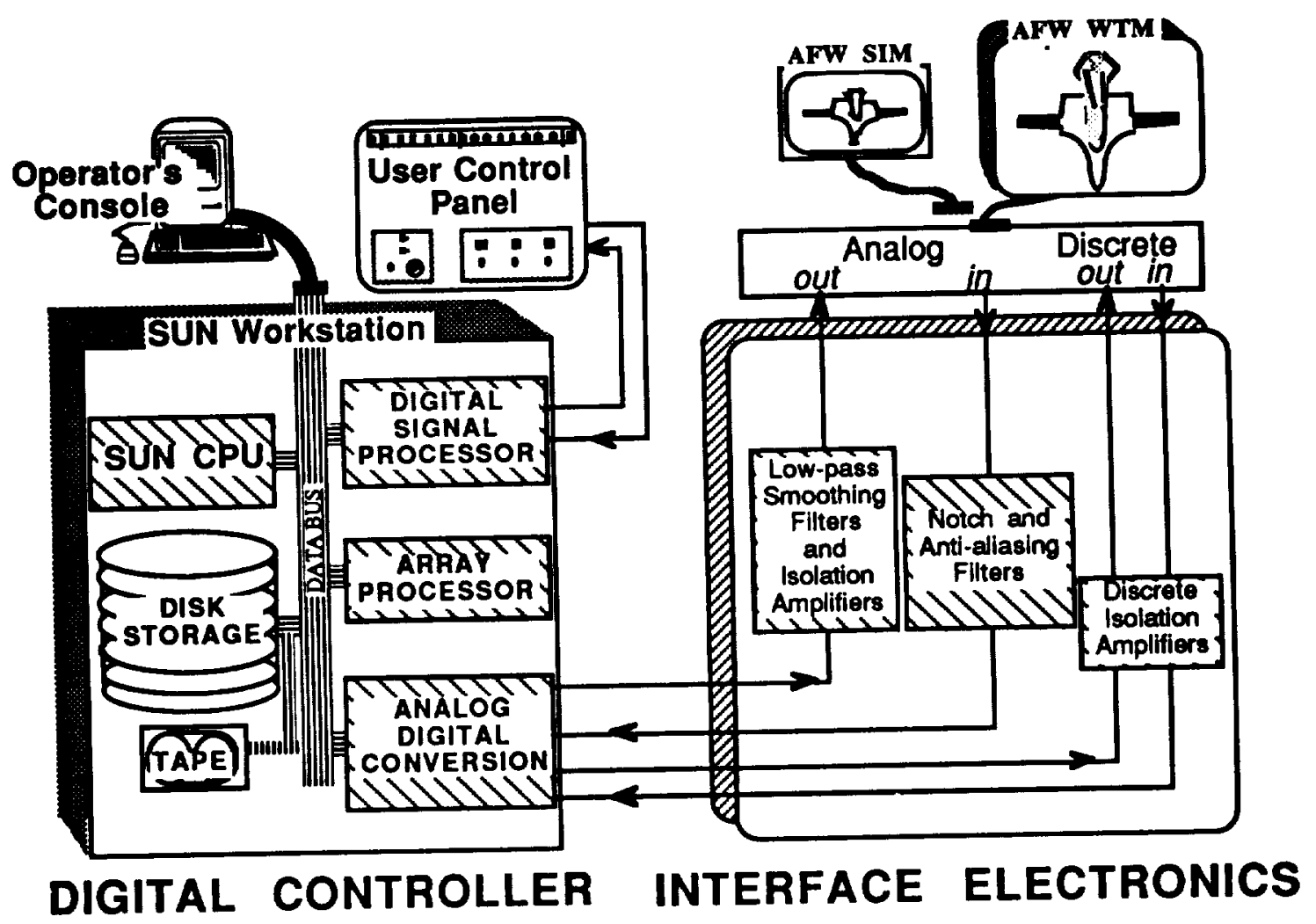

Figure 6. - Schematic of digital controller and interface electronics

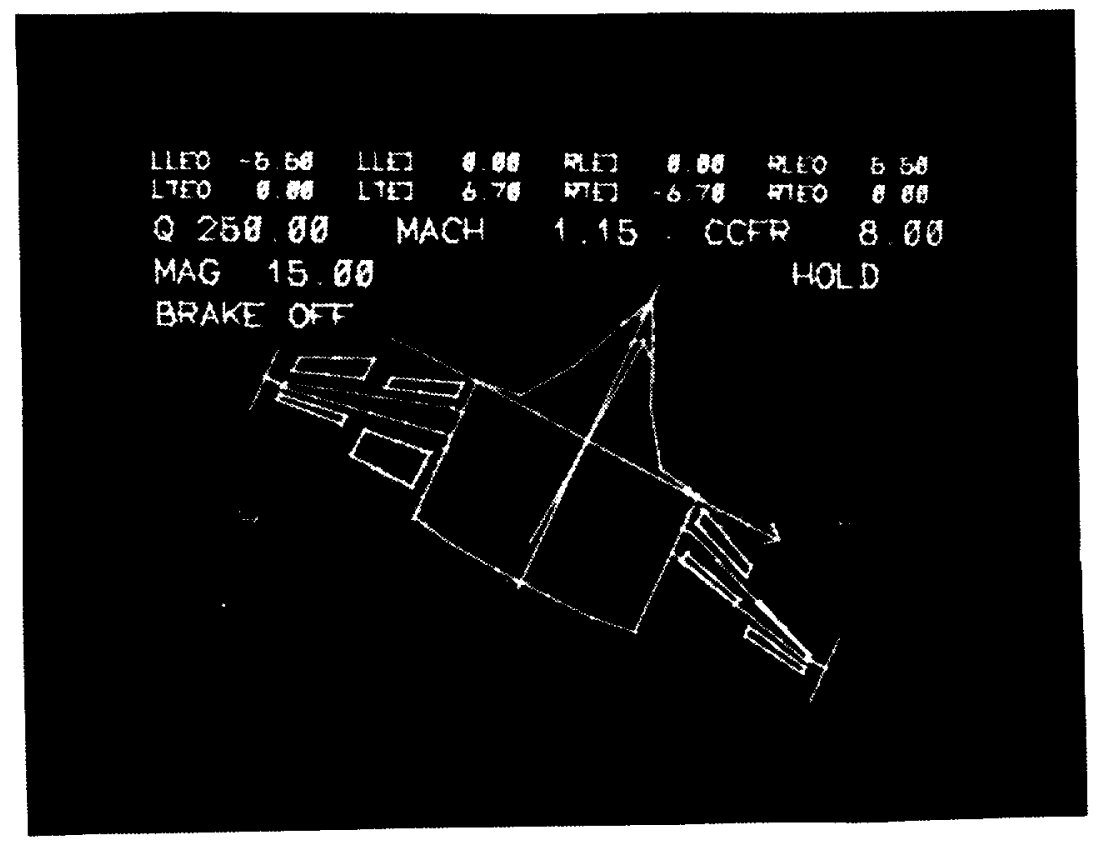

Figure 7. - Wireframe oulline of AFW model used in hot bench simulation 

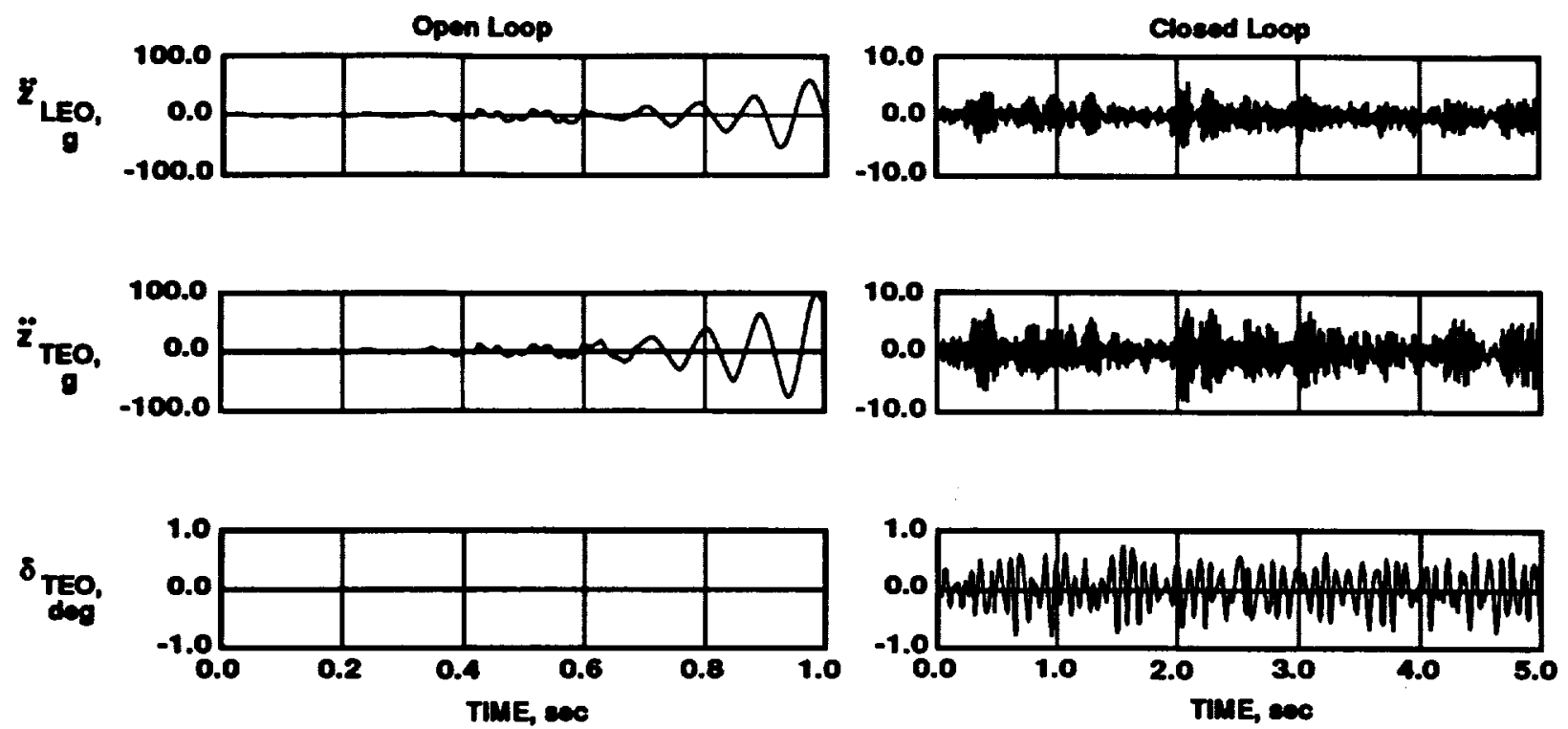

Figure 8. - Open- and closed-loop symmetric responses from nonlinear batch simulation, Dynamic pressure $=300 \mathrm{psf}$, Disturbance quantity $=1 \mathrm{f} / \mathrm{s}$ ms Dryden gust

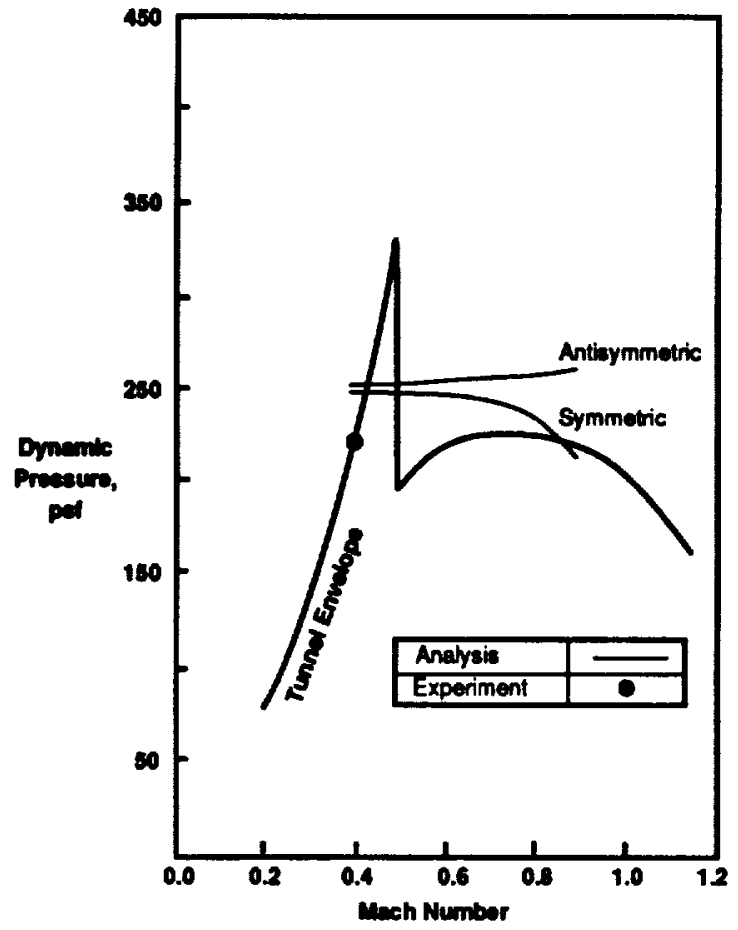

Figure 9. - TDT operating envelope in air with predicted and measured open-loop flutter point,

Tip ballast store coupled 


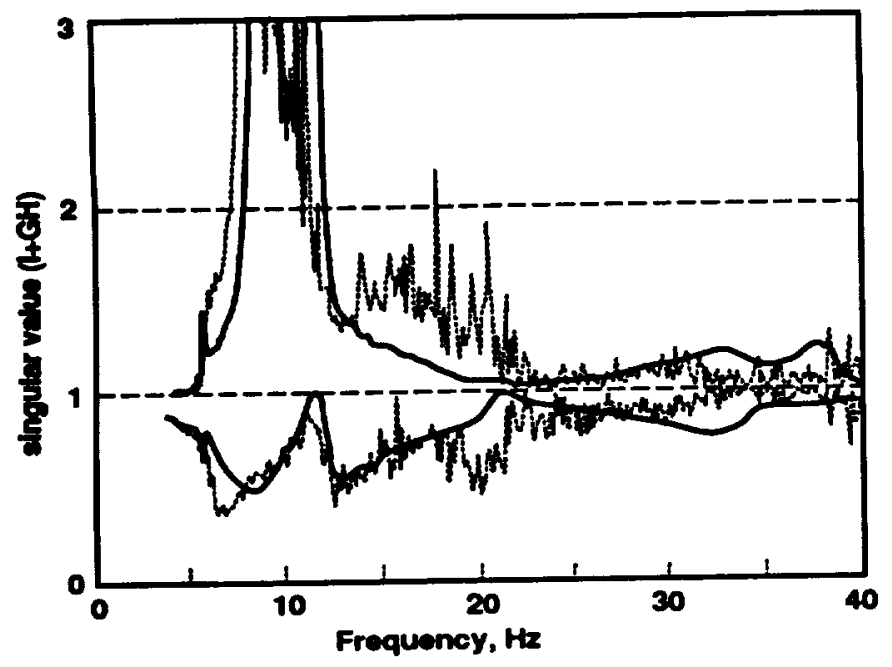

(a) Singular values at plant output

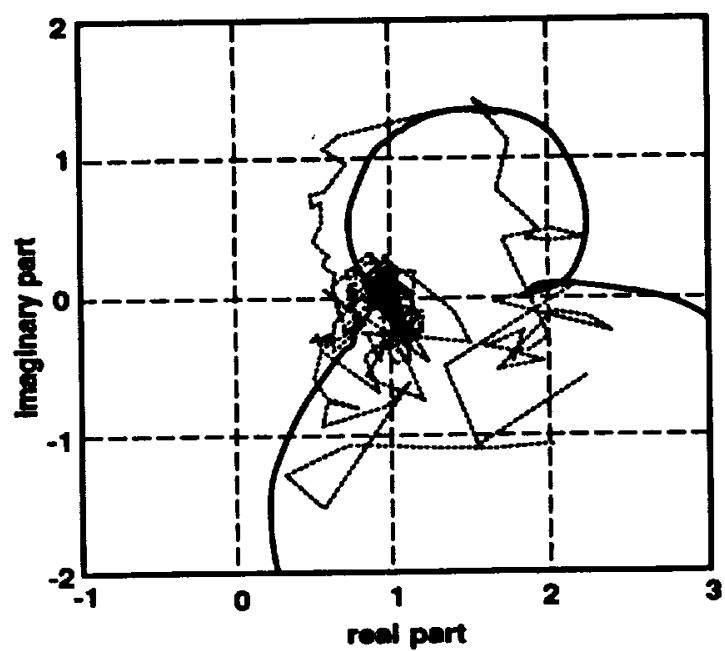

(b) Determinant at plant output

Figure 10. - Theoretical and experimental controller performance evaluation results, Symmetric portion of Control Law 1, Dynamic pressure $=200 \mathrm{psf}$

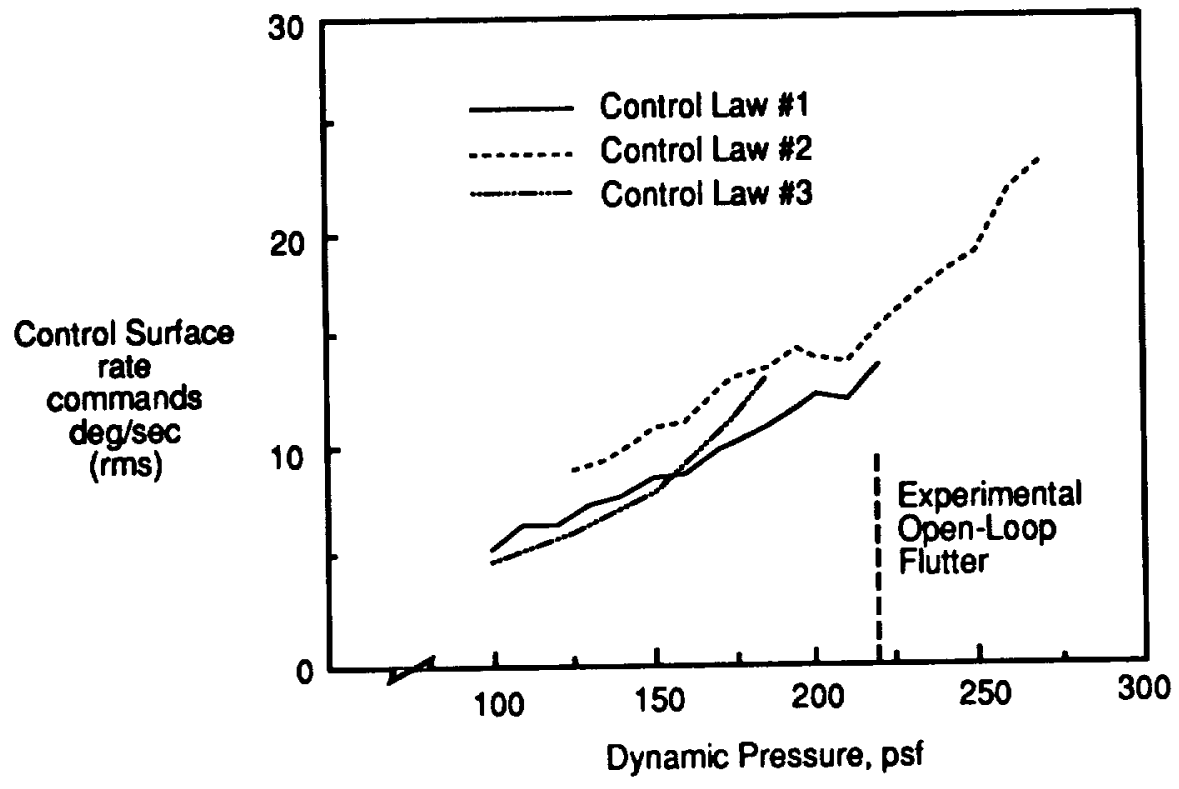

Figure 11. - Control-surface rate commands 


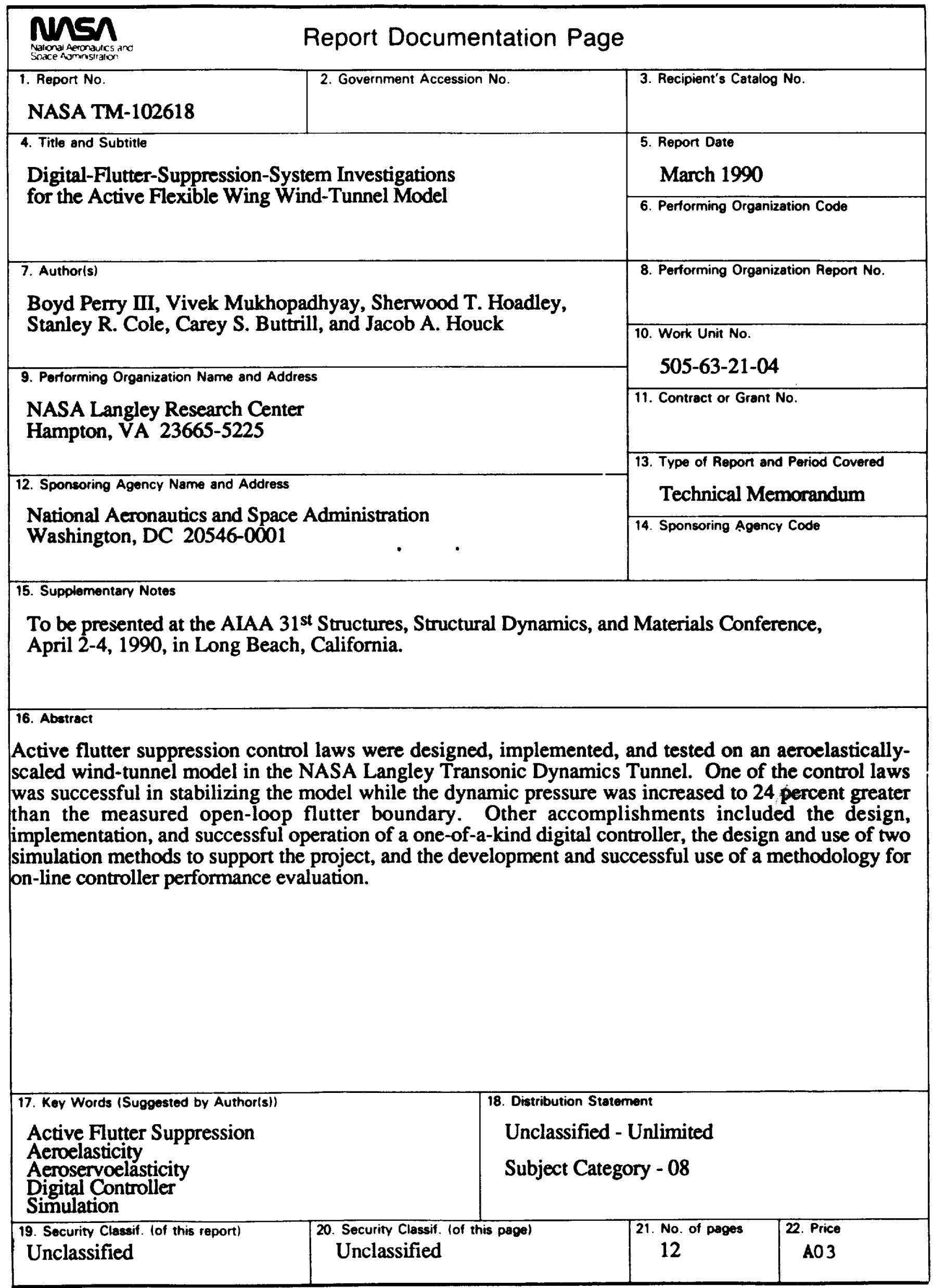



$=$ 